\title{
Atheromatous Plaque in the Distal Aortic Arch Creating the Potential for Cerebral Embolism During Cardiopulmonary Bypass
}

\author{
Kimikazu Hamano, MD; Yoshitaka Ikeda, MD; Akihito Mikamo, MD; \\ Haruhiko Okada, MD; Hidenori Gohra, MD; Nobuya Zempo, MD; \\ Kayo Ueda, MD*; Kazumi Kimura, MD*; Kazuya Murata, MD*; \\ Masunori Matsuzaki, MD*; Kensuke Esato, MD
}

\begin{abstract}
The present study evaluated the risk in cardiac patients of rupture of a plaque by a jet stream from the arch cannula. The entire thoracic aorta and cardiac function were routinely monitored by transesophageal echocardiography (TEE) in 88 adult patients who underwent coronary artery bypass surgery. The changes in the atheromatous plaque in the distal aortic arch were observed before and after cardiopulmonary bypass. Of the 88 patients, 13 were found to have preoperative atheromatous plaque at the distal aortic arch and $8(61.5 \%)$ of them suffered plaque rupture caused by jet stream from the arch cannula. Only 1 patient experienced apparent embolic episodes manifesting as cerebral and left leg embolisms; the remaining 7 had no clinical embolic symptoms. In order to prevent atheroembolic events, attention should be paid not only to the ascending aorta, but also to the distal arch and in this regard TEE is useful for detecting atheromatous changes of the aorta. (Jpn Circ J 2001; 65: 161164)
\end{abstract}

Key Words: Atheroma plaque; Cardiopulmonary bypass; Coronary artery bypass surgery; Embolism; Transesophageal echocardiography

C erebral embolism remains one of the most serious complications following cardiopulmonary bypass (CPB). Atherosclerosis of the ascending aorta is associated with a high perioperative risk of stroke after cardiac surgery ${ }^{1,2}$ and there are various measures ${ }^{3-6}$ to prevent atheroembolic complications during these operations. Most studies of the atheromatous changes of the aorta have focused on the ascending aorta where the arch cannula is inserted and the aortic cross clamp applied. The thoracic aorta, especially at the site just distal to the left subclavian artery, which we have defined as the "distal aortic arch', has the highest incidence of atherosclerotic aneurysm. We have recently been investigating the influence of the jet stream from the arch cannula routinely used in $\mathrm{CPB}$ and the aim of the present study was to clarify the relationship between the atherosclerotic changes of the distal aortic arch and atheroembolic events.

\section{Methods}

\section{Patients}

A total of 88 consecutive adult patients who underwent coronary artery bypass surgery were enrolled (66 men, 22 women; age range, 42-83 years, mean age, 63.2 years). Cardiac function was routinely monitored by transesophageal echocardiography (TEE) during surgery in all patients.

(Received August 16, 2000; revised manuscript received November 24, 2000; accepted November 29, 2000)

First Department of Surgery and *Second Department of Internal Medicine, Yamaguchi University School of Medicine, Yamaguchi, Japan

Mailing address: Kimikazu Hamano, MD, First Department of Surgery, Yamaguchi University School of Medicine, 1-1-1 Minam-Kogushi, Ube, Yamaguchi 755-8505, Japan
A Sarns 5.2-mm or 6.5-mm arch cannula (3M Health Care, Ann Arbor, MI, USA) was used in all patients except one. There were no patients in whom the arch cannula was unable to be used because of atheromatous plaque and/or calcification of the ascending aorta. For the CPB circuit, a centrifugal pump (Bio-Console; Bio-Medicus Inc, Eden Prairie, MN, USA) with a perfusion index of $2.4 \mathrm{~L} \cdot \mathrm{min}^{-1}$. $\mathrm{m}^{-2}$ body surface area, was employed in all the patients except for one who had cerebral arterial disease and in whom pulsatile perfusion was applied with a roller pump. Mild hypothermia, with a rectal temperature between $31^{\circ} \mathrm{C}$ and $33^{\circ} \mathrm{C}$, was maintained during cardiac arrest.

\section{Transesophageal Echocardiography (TEE)}

In our hospital, TEE (SSD-2200; Aloka Co, Japan) is performed by a cardiologist for every adult patient during cardiac surgery. Before and after CPB, not only the cardiac area, but the entire thoracic aorta is observed to identify any functional or anatomical changes. A multiplane echo probe is inserted after the induction of anesthesia. To assess the severity of atherosis semiquantitatively, we classified atheromatous lesions of the distal aortic arch into 4 categories: normal intima, intimal thickening, atheroma, and complicated lesion?,8 A normal intima was indicated by a fine and smooth intima-media complex echo, visualized at the center of the sector where the ultrasonic beam was perpendicular to the aortic wall. Intimal thickening was indicated by diffuse intimal thickening that produced irregular and dense echoes around the surface of the intima without raised plaque. Atheroma was indicated by the presence of significant raised plaque or a cystic lesion. A complicated lesion was ulcer formation and calcified plaque that produced a marked 
Table 1 Cases Having Plaque Rupture After Cardiopulmonary Bypass

\begin{tabular}{ccccc}
\hline \hline Case no. & Age (years) & Sex & Procedure & Clinical embolic episode \\
\hline 1 & 74 & Male & CABG & + (Cerebral) (Left leg) \\
2 & 49 & Male & CABG & - \\
3 & 77 & Female & CABG & - \\
4 & 67 & Male & CABG & - \\
5 & 67 & Male & CABG & - \\
6 & 61 & Male & CABG & - \\
7 & 72 & Male & CABG & - \\
8 & 83 & Male & CABG & - \\
\hline
\end{tabular}

$C A B G$, coronary artery bypass grafting.

increase in echo intensity together with acoustic shadowing behind the lesion. Although it is well known that calcification of the ascending aorta is a risk factor of embolism, calcification itself does not seem to be a risk factor in the descending aorta 1,2 We considered that the atheromatous plaque in the descending aorta might be a major risk factor for embolism created by the jet from the arch cannula. Only atheromatous plaque was considered 'abnormal' in this study.

\section{Results}

Of the 88 patients, $13(14.8 \%)$ were found preoperatively to have atheromatous plaque at the level of the distal aortic arch (10 men, 3 women; age range, $47-83$ years, mean age, 67 years). An arch cannula was used in all patients because of the possibility of arteriosclerotic change in the iliofemoral region, which might have led to retrograde atheroembolism. Of these 13 patients, $8(61.5 \%)$ suffered atheromatous plaque rupture caused by the jet stream of blood from the arch cannula after CPB (Table 1); 3 of them

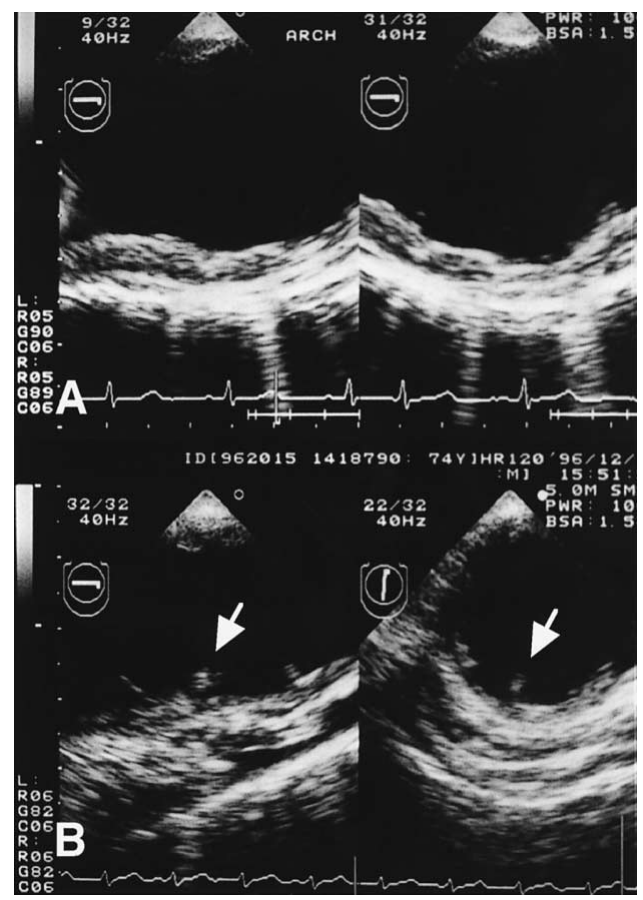

Fig 1. TEE findings before and after cardiopulmonary bypass (CPB) in case 1. Moderate atheromatous plaque was detected before $\mathrm{CPB}$ (A) at the site of the distal aortic arch. After CPB, the plaque was ruptured by a jet stream from the arch cannula (B; arrow). had severe rupture, termed the 'sandblast effect', with 2 different outcomes.

\section{Case 1}

A 74-year-old man underwent coronary artery bypass grafting (CABG) for 2-vessel disease. Moderate atheromatous plaque had been detected by TEE prior to CPB. Because arteriosclerosis of the ilio-femoral artery existed, an arch cannula was selected in preference to a femoral cannula. After CPB, TEE revealed the rupture of the plaque in the distal aortic arch (Fig 1). His left leg became cyanotic and an emergency angiogram showed occlusion of the left posterior tibial artery (Fig 2A). Cerebral infarction was subsequently confirmed by brain computed tomography (CT) (Fig 2B). It is possible that multiple embolization occurred with only 2 clinical embolic episodes.

\section{Case 2}

A 49-year-old man who had received a renal transplant at the age of 32 underwent CABG. He was discovered to have atheromatous plaque in the ilio-femoral region, and because the transplanted kidney was in the right iliac position, it seemed preferable to use an arch cannula rather than a femoral cannula. Although severe rupture of the atheromatous plaque was confirmed by TEE after CPB (Fig 3), as in case 1 , he suffered no clinical signs of embolism; however, he might have had latent emboli in some organs.

\section{Case 3}

A 77-year-old woman underwent CABG for 3-vessel disease. Moderate to severe atheromatous plaque had been detected by TEE prior to CPB. Because of our previous experiences of plaque rupture, an arch cannula with lateral windows (Sarns soft flow; 3M Health Care, Ann Arbor, MI, USA) was selected in preference to the usual arch cannula. After CPB, TEE revealed the rupture of the plaque in the distal aortic arch, but without clinical embolic episode, as in case 2 .

The remaining patients who had minor plaque rupture did not suffer any clinical embolic episodes.

\section{Discussion}

The majority of the studies of the atheromatous changes of the aorta have focused on the ascending aorta rather than on the distal aortic arch. Many have reported the relationship between the severity of the atheromatous changes of the aorta and episodes of cerebral embolism, 1,2 so cardiac surgeons try to prevent the occurrence of embolism associated with aortic clamping and insertion of the arch cannula3-6 Hartman et $\mathrm{al}^{9}$ investigated the relationship between the degree of atherosclerosis and the incidence of stroke. In 

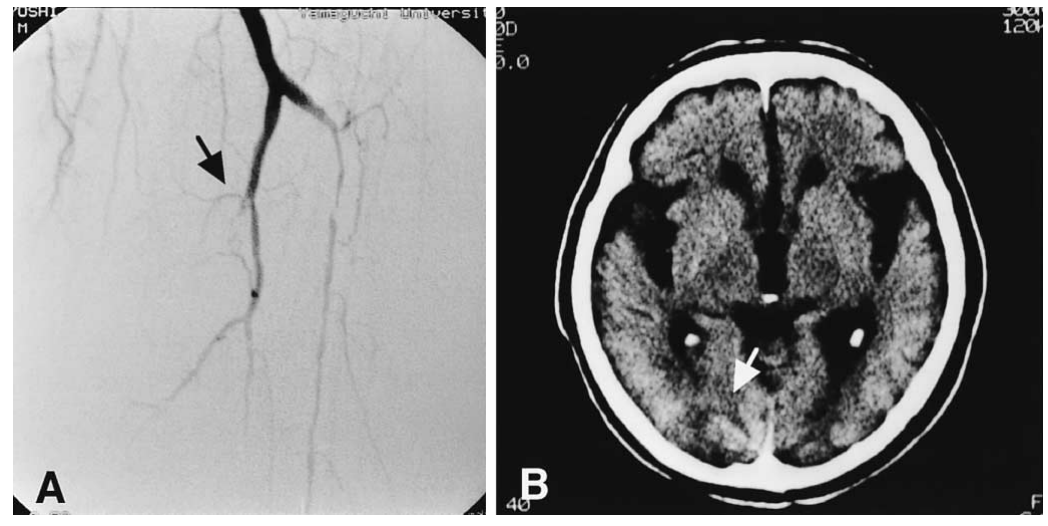

Fig 2. Angiogram of the left lower limb and brain $\mathrm{CT}$ in case 1 . The angiogram showed occlusion of the left posterior tibial artery (A; arrow) and $\mathrm{CT}$ revealed cerebral infarction (B; arrow).

patients with severe calcification of the ascending aorta, the so called 'porcelain aorta', a clamp should not be applied in order to prevent rupture of the calcified aortic wall, which would lead to aortic dissection and atheroembolization.-6 Generally the cardiac surgeon palpates the ascending aorta to identify whether a calcified area exists, and in some institutes, an echo probe has been used under direct vision, known as epiaortic echo. When seriously calcified or atheromatous areas exist, a clamp and/or a cannula must be applied to an area where either no lesion or only a mild lesion exists.

Vaduganathan et al estimated that there was a $73 \%$ exact agreement between TEE and the pathologic grading and that discrepancies were mostly attributable to the inability of TEE to detect superficial ulceration ${ }^{10}$ Hartman et al demonstrated a uniformly high agreement for the interpretation of TEE, which indicates the excellent reproducibility of TEE grading and stratification of aortic atheroma! 1 Konstadt et al showed that the negative predictive value of the TEE was $100 \%$, with a positive predictive value of $34 \%$, and recommended that if the TEE examination was positive for plaque, epiaortic scanning should be considered? Davila-Roman and colleagues reported that biplane TEE significantly underestimated the severity of ascending aortic atherosclerosis, compared with epiaortic ultrasound.2 It should be borne in mind that palpation of the ascending aorta significantly underestimates the presence and severity of atherosclerosis, when compared with epiaortic ultrasound and biplane TEE. From this study and the literatures ${ }^{7-9}$ severely raised atheroma with an irregular surface, or with cystic or heterogenous contents, are the most easily ruptured.

D'Agostino et al reported the usefulness of screening carotid stenosis by ultrasonography for identifying patients at high risk of postoperative stroke! ${ }^{3}$ Although the incidence of carotid artery stenosis is low in Japan, stenosis of the intracranial artery is relatively common, the diagnosis of which may be more effectively made by magnetic resonance angiogram. We have recently been screening intracranial arterial stenosis by magnetic resonance angiography, which has revealed critical stenosis and occlusion of the major cerebral arteries in some patients. Only one patient in the present study had a carotid stenosis, and endoarterectomy was performed safely in conjunction with CABG.

The best strategy for patients with atheromatous plaque at the distal aortic arch is still controversial. The possible alternative cannulation sites for such patients are the proximal descending aorta and the axillary or femoral arteries. Axillary arterial perfusion still carries the risk of bursting the atheroma, and femoral perfusion is associated with the

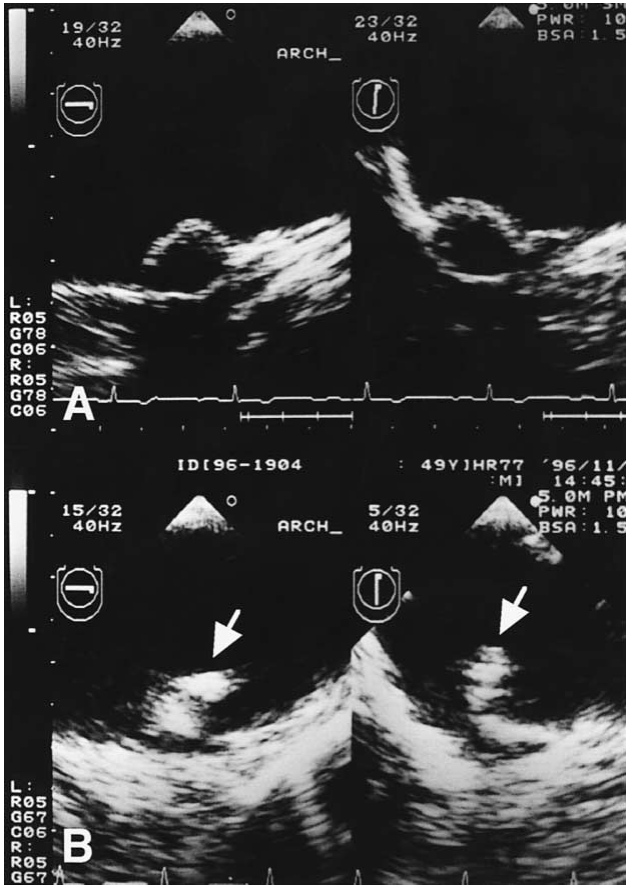

Fig 3. Transesophageal echocardiography (TEE) findings before and after cardiopulmonary bypass (CPB) in case 2 . Moderate atheromatous plaque before $\mathrm{CPB}(\mathrm{A})$ ruptured severely after $\mathrm{CPB}$ as in case 1 (B; arrow). This patient did not suffer a clinical embolic episode.

risk of emboli arising from atheroma in the ilio-femoral region. Grossi and coworkers measured the flow in the aortic arch during CPB using 2 types of arch cannula and revealed that the peak forward aortic flow velocity with a short (1.5 $\mathrm{cm}$ ) cannula was significantly greater than that with a long $(7.0 \mathrm{~cm})$ cannula 14 Furthermore, severe turbulence in the aortic arch was found when the short cannula was used, whereas only mild or no turbulence resulted when the long cannula was used. Groom et al performed in vitro velocimetry measurements during perfusion with 5 aortic cannulae at the exit site within a cast of a human aorta attached to a mock circulatory loop! $!^{15}$ They demonstrated that the cannula designed with lateral windows and a dispersing cone produced a lower velocity and less concentrated flow pattern at the cannula exit and warned that the use of pulsatile flow doubled the velocity at the cannula exit, which could present a higher risk of atheroembolism generation in patients with atherosclerotic disease in the ascending aorta. 
Unfortunately, despite using a cannula with lateral windows for the patient who had atheromatous plaque in the distal aorta (Case 3 ), we could not prevent the plaque rupture. In theory, this cannula should be useful for the patients with mild atheromatous plaque.

For patients with severe atheromatous plaque, minimally invasive direct coronary artery bypass or CABG without CPB is the only way to prevent embolization. The disadvantages of this surgery are the difficulty in achieving complete revascularization and the quality of anastomosis. However, the prevention of embolism is definitely worthwhile compared with those disadvantages.

Singh and associates reported that the risk factors for stroke were an age greater than 70 years, severity of aortic arch atherosclerosis, and severe hypotension in the perioperative period ${ }^{16}$ They also found that there was not a higher incidence of overt neurologic injuries with normothermic perfusion than with hypothermic perfusion. Marschall et al found that only severe arch disease correlated significantly with stroke! According to their series, severe aortic disease was not present in patients aged less than 50 years, but it was found in approximately $20 \%$ of those aged over 70 years. These data suggest that we should screen the aorta by TEE and/or epiaortic echo at least in patients aged over 70 years old; however, in our study, we found 2 patients younger than 50 years old who had moderate atheromatous plaque at the distal aortic arch. Thus, it may be advisable to monitor the aorta in all patients who undergo cardiac surgery.

In conclusion, we recommend monitoring not only the ascending aorta, but also the distal aortic arch to prevent atheroembolism during CPB. TEE is a useful method for detecting the atheromatous changes of the aorta. To prevent embolism the use of a cannula with a lateral window might be useful for the patients with mild athromatous plaque, but for the patients with moderate or severe atheromotous plaque, off pump CABG is only the way to prevent embolism.

\section{References}

1. Marschall K, Kanchuger M, Kssler K, Grossi E, Varmush L, Roggen $\mathrm{S}$, et al: Superiority of transesophageal echocardiography in detecting aortic arch atheromatous disease: Identification of patients at increased risk of stroke during cardiac surgery. J Cardiothorac Vasc Anesth 1994; 8: 5-13

2. Konstadt SN, Reich DL, Kahn R, Viggiani RF: Transesophageal echocardiography can be used to screen for ascending aortic atherosclerosis. Anesth Analg 1995; 81: 225-228

3. Trehan N, Mishra M, Dhole S, Mishra A, Karlekar A, Kohli VM: Significantly reduced incidence of stroke during coronary artery bypass grafting using transesophageal echocardiography. Eur $J$ Cardiothorac Surg 1997; 11: 234-242

4. Suma H: Coronary artery bypass grafting in patients with calcified ascending aorta: Aortic no-touch technique. Ann Thorac Surg 1989; 48: $728-730$

5. Peigh PS, DiSesa VJ, Collins JJ, Cohn LH: Coronary bypass grafting with totally calcified or acutely dissected ascending aorta. Ann Thorac Surg 1991; 51: $102-104$

6. Accola KD, Jones EL: Coronary revascularization in a patient with porcelain aorta and cacified great vessels. Ann Thorac Surg 1993; 55: $514-515$

7. Matsuzaki M, Ono S, Tomochika Y, Michishige H, Tanaka N, Okuda $\mathrm{F}$, et al: Advances in transesophageal echocardiography for the evaluation of atherosclerotic lesions in thoracic aorta: The effect of hypertension, hypercholesterolemia, and aging on atherosclerotic lesions. Jpn Circ J 1992; 56: 592-602

8. Tomochika Y, Tanaka N, Ono S, Murata K, Muro A, Yamamura T, et al: Assessment by transesophageal echography of atherosclerosis of the descending thoracic aorta in patients with hypercholesterolemia. Am J Cardiol 1999; 83: 703-709

9. Hartman GS, Yao FS, Bruefach M III, Barbut D, Peterson JC, Purcell $\mathrm{MH}$, et al: Severity of aortic atheromatous disease diagnosed by transesophageal echocardiography predicts stroke and other outcomes associated with coronary artery surgery: A prospective study. Anesth Analg 1996; 83: 701-708

10. Vaduganathan P, Ewton A, Nagueh SF, Weilbaecher DG, Safi HJ, Zoghbi WA: Pathologic correlates of aortic plaques, thrombi and mobile 'aortic debris' imaged in vivo with transesophageal echocardiography. J Am Coll Cardiol 1997; 30: 357-363

11. Hartman GS, Peterson J, Konstadt SN, Hahn R, Szatrowski TP, Charlson ME, et al: High reproducibility in the interpretation of intraoperative transesophageal echocardiographic evaluation of aortic atheromatous disease. Anesth Analg 1996; 82: 539-543

12. Davila-Roman VG, Phillips KJ, Daily BB, Davila RM, Kouchoukos NT, Barzilai B: Intraoperative transesophageal echocardiography and epiaortic ultrasound for assessment of atherosclerosis of the thoracic aorta. J Am Coll Cardiol 1996; 28: 942-947

13. D'Agostino RS, Svensson LG, Neumann DJ, Balkhy HH, Williamson WA, Shahian DM: Screening carotid ultrasonography and risk factors for stroke in coronary artery surgery patients. Ann Thorac Surg 1996; 62: $1714-1723$

14. Grossi EA, Kanchuger MS, Schwartz DS, Mcloughlin DE, LeBoutillier M III, Ribakove GH, et al: Effect of cannula length on aortic arch flow: Protection of the atheromatous aortic arch. Ann Thorac Surg 1995; 59: 710-712

15. Groom RC, Hill AG, Kuban B, O'Neill W, Akl BF, Speir AM, et al: Aortic cannula velocimetry. Perfusion 1995; 10: $183-188$

16. Singh AK, Bert AA, Feng WC, Rotenberg FA: Stroke during coronary artery bypass grafting using hypothermic versus normothermic perfusion. Ann Thorac Surg 1995; 59: 84-89 